\title{
BMJ Open Dural ossification associated with ossification of ligamentum flavum in the thoracic spine: a retrospective analysis
}

\author{
Bo Li, ${ }^{1}$ Guixing Qiu, ${ }^{1}$ Shigong Guo, ${ }^{2}$ Wenjing Li, ${ }^{1}$ Ye Li, ${ }^{1}$ Huiming Peng, ${ }^{1}$ \\ Chu Wang, ${ }^{1}$ Yu Zhao ${ }^{1}$
}

To cite: Li B, Qiu G, Guo S, et al. Dural ossification associated with ossification of ligamentum flavum in the thoracic spine: a retrospective analysis. BMJ Open 2016;6: e013887. doi:10.1136/ bmjopen-2016-013887

- Prepublication history for this paper is available online. To view these files please visit the journal online (http://dx.doi.org/10.1136/ bmjopen-2016-013887).

Investigation performed at the Department of Orthopaedic Surgery, Peking Union Medical College Hospital, Chinese Academy of Medical Sciences and Peking Union Medical College, Beijing, China.

Received 15 August 2016 Revised 4 November 2016 Accepted 24 November 2016

CrossMark

For numbered affiliations see end of article.

Correspondence to

Dr Yu Zhao;

zhaoyupumch@126.com

\section{ABSTRACT}

Objectives: To investigate the incidence, distribution and radiological characteristics of dural ossification (DO) associated with ossification of ligamentum flavum (OLF) in the thoracic spine.

Design: A retrospective radiographical analysis.

Setting: This study was conducted at a single institution in China.

Participants: 53 patients with OLF who underwent posterior decompression surgery between January 2011 and July 2015 in a single institution were enrolled in this study. The decompression segments were grouped according to imaging evaluation and intraoperative evidences.

Outcome measures: The demographic distribution, radiological data and detailed surgical records were collected. First, preoperative CT images of decompressed segments were evaluated to identify imaging signs of DO. The 'tram tack sign' (TTS), 'comma sign' and 'bridge sign' were considered as characteristic imaging findings of DO in OLF. 4 kinds of confusing signs (false TTS) were identified and excluded. Then detailed surgical records were reviewed to finally identify segments with DO.

Results: The incidence of DO in patients with OLF was $43.4 \%$. The incidence of DO in OLF segments was $21.5 \%$. OLF was more common in the lower thoracic spine, and more than half $(53.8 \%)$ of the DO was located in T9-T12. TTS was the most common sign, but it might be misdiagnosed. After excluding 4 kinds of false TTS, the sensitivity and specificity of imaging diagnosis were $94.23 \%$ and $94.21 \%$, respectively. Conclusions: DO was relatively common in thoracic OLF, especially in T9-T12. TTS might be misdiagnosed. After excluding 4 kinds of false TTS, the accuracy of imaging diagnosis was relatively high.

\section{INTRODUCTION}

Ossification of ligamentum flavum (OLF) is the most common cause of thoracic spinal stenosis, ${ }^{1-3}$ especially in East Asian populations. With the progression of OLF, the

\section{Strengths and limitations of this study}

- This study made up for the current lack of understanding of dural ossification (D0) associated with ossification of ligamentum flavum (OLF) in the thoracic spine.

- The incidence, distribution and radiological characteristics of DO were described and analysed in detail.

- The study increased the accuracy of identifying DO in OLF before surgery.

- The study population was limited to patients with OLF who need surgical treatment.

spinal cord will be subjected to severe compression and the patient may eventually be paralysed. Surgical treatment is generally accepted as the best option. ${ }^{2-6}$ When compressed, the dura mater may also ossify. Then the ossified ligamentum flavum and dura mater fuse to be one inseparable bony tissue. This will increase the difficulty of surgery and the risk of spinal cord injury and cerebrospinal fluid leakage. ${ }^{78}$ Preoperative identification of dural ossification (DO) can help surgeons to adopt appropriate surgical technique and get prepared to deal with the intraoperative dural laceration. In a preliminary study, the 'tram track sign' (TTS) and 'comma sign' (CS) were reported to be associated with DO. ${ }^{9}$ However, owing to the relatively small study population and lack of awareness of these specific image signs accurate diagnosis of DO could not be obtained in previous studies. ${ }^{2}{ }^{10}$ Since the incidence of OLF and DO is relatively low, few studies have focused on this issue. ${ }^{2} 910$ The incidence and distribution of DO were also unclear. The present study aimed to investigate the incidence, distribution and radiological characteristics of DO in thoracic OLF. 


\section{METHODS}

\section{Study population}

Patients with thoracic OLF who underwent posterior decompression surgery between January 2011 and July 2015 in a single Chinese institution were enrolled in this retrospective cohort study. The exclusion criteria were patients with OLF with thoracic trauma, infection, tumour and deformity. Fifty-three cases were finally included in this study, with Institutional Review Board approval. The main symptoms included numbness, zonaesthesia, asthenia, gait disturbance, back pain and bladder/bowel dysfunction. The indication of surgical treatment was neurological deterioration. All patients underwent open posterior decompression. Intraoperative, after resecting the lamina, the ossified ligamentum flavum was lifted and separated from the dura mater by gentle dissection. If ossifying, the dura mater will fuse with the ossified ligamentum flavum, which makes the separation impossible. In this condition, the ossified dura mater was also resected. Clinical data, preoperative CT and detailed surgical records were available for all the included patients. The decompression levels were grouped according to imaging evaluation and intraoperative evidences described below.

\section{Imaging evaluation and identification of DO}

Preoperative axial CT scans of surgical decompression segments (involving vertebral levels) were the primary imaging to be assessed for every included patient. In our preliminary screening study, the TTS, CS and 'bridge sign' (BS) were considered as characteristic imaging findings of DO in OLF (figure 1). TTS and CS have been reported before. TTS was defined as a hyperdense bony excrescence, with a hypodense centre (figure 1A, D). It consisted of three parts in the axial CT scan: ossified dura mater (hyperdense), ossified ligamentum flavum (hyperdense) and the space between them (hypodense). These three parts together formed a tram track-like shape. CS was an imaging manifestation of ossification of one-half of the dura mater (figure $1 \mathrm{~B}, \mathrm{E}$ ). Ossified dura mater and ligamentum flavum fused to be the head of the 'comma'. The tail of the 'comma' indicated that ossification extended to the lateral or ventral dura mater. BS, which was first reported in this study, was a hyperdense bony connection of bilateral OLF (figure 1C, F). The dorsal ossified dura mater presented as a bridge between bilateral ossified ligamentum flavum. Segments with the above signs on axial CT images would be initially diagnosed with DO in OLF.

TTS might be misdiagnosed as found in our preliminary screening study. So we defined and excluded four kinds of confusing signs (false TTS; figure 2): a false TTS consisted of a facet joint (figure 2A); a false TTS consisted of an ossified ligamentum flavum and superior articular process (figure 2B); a false TTS between the ossified ligamentum flavum and lamina (figure 2C); and a false TTS between the inner and outer bone cortex of a superior articular process (figure 2D). For atypical signs reference to adjacent imaging of the same segment might be helpful (figure 2E).

To ensure reliability, imaging evaluation was performed before the review of surgical records. Two observers, blind to the groups, were responsible for the imaging evaluation. One was responsible for our preliminary screening study, who defined the TTS, CS, BS and four
Figure 1 Special imaging findings of DO. (A, D) Tram track sign (A: bilateral, $D$ : unilateral): a hyperdense bony excrescence, with a hypodense centre. TTS consisted of three parts: ossified dura mater (hyperdense), ossified ligamentum flavum (hyperdense) and the space between them (hypodense). (B, E) Comma sign: ossification of one-half of the dura mater. Ossified dura mater and ligamentum flavum fused to be the head of the 'comma'. The tail of the 'comma' indicated that ossification extended to the lateral or ventral dura mater. (C, F) Bridge sign: a hyperdense bony connection of bilateral OLF. Dorsal ossified dura mater presented as a bridge between bilateral ossified ligamentum flavum. DO, dural ossification; OLF, ossification of ligamentum flavum.
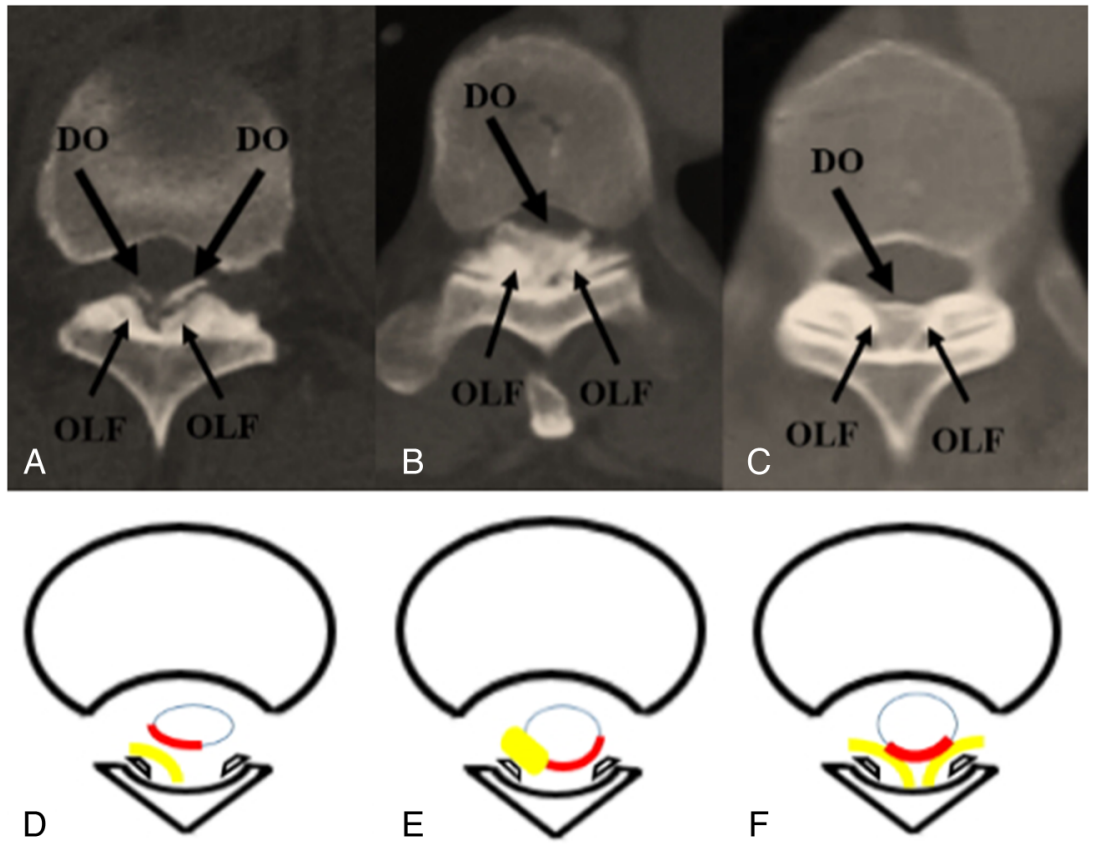
Figure 2 Four kinds of confusing signs (false TTS) and analysis of adjacent levels: (A) a false TTS of the facet joint; (B) a false TTS of the ossified ligamentum flavum and superior articular process; (C) a false TTS between the ossified ligamentum flavum and lamina; (D) a false TTS between the inner and outer bone cortex of the superior articular process. (E) Imaging E2 was adjacent level of the same segment with E1. Adjacent imaging (E2) could help to identify the imaging findings in E1 was false TTS of ossified ligamentum flavum and superior articular process. OLF, ossification of ligamentum flavum; TTS, tram track sign.

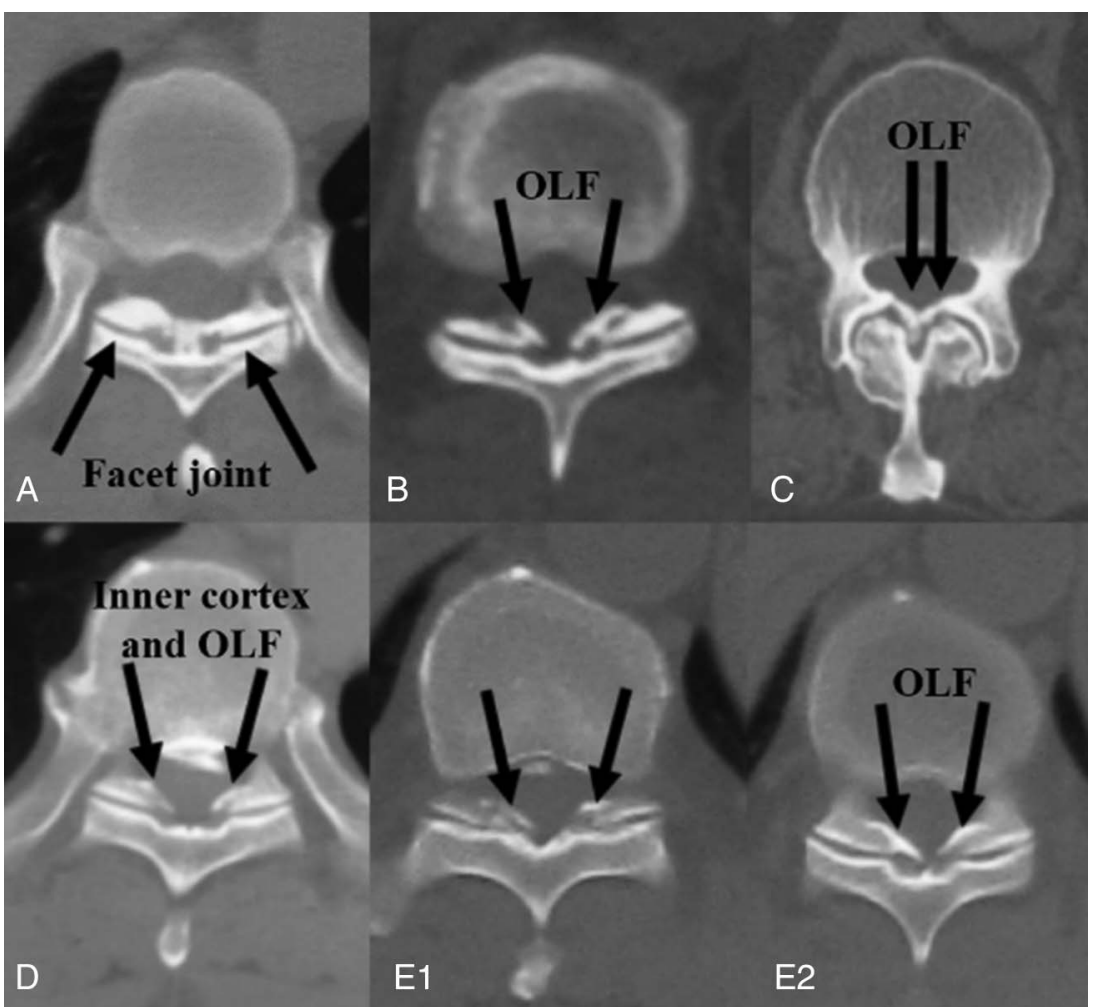

kinds of confusing signs (false TTS). The other was responsible for our diagnosis study using the defined criteria of different kinds of imaging signs. Surgical decompression segments would then be divided into the following three categories (initial diagnosis): non-OLF segments, OLF segments without DO and OLF segments with DO. After imaging evaluation, surgical records were reviewed to identify which segments were truly associated with DO. This would be regarded as the final diagnosis. The incidence and distribution of DO were recorded.

\section{Statistical analyses}

Initial diagnosis was compared with the final diagnosis for calculating the sensitivity and specificity of imaging diagnosis. The test accuracy, positive and negative predictive values were also calculated. Data were presented as mean \pm SD. SPSS software (V.20.0, Chicago, Illinois, USA) was used for statistical analysis.

\section{RESULTS}

\section{Population}

Fifty-three patients with OLF (26 female; age 53.87 \pm 10.42 , range $30-74$ ) were included in this retrospective study. Twenty-three cases (13 female) had intraoperative evidence of DO. The mean age of the DO group was $54.65 \pm 9.62$ compared with $53.27 \pm 11.12$ in the non-DO group ( $\mathrm{p}>0.05$; table 1 ).

\section{Incidence}

Of the 53 included patients, 242 surgical decompression segments were diagnosed with OLF by axial CT scan.

\begin{tabular}{llll} 
Table 1 & Basic information of included patients with OLF \\
\hline & $\begin{array}{l}\text { OLF with } \\
\text { DO }\end{array}$ & $\begin{array}{l}\text { OLF without } \\
\text { DO }\end{array}$ & Total \\
\hline Cases & 23 & 30 & 53 \\
Sex (female/ & $13 / 10$ & $13 / 17$ & $26 / 27$ \\
male) & & & \\
Age (years) & 54.65 & $53.27 \pm 11.12$ & 53.87 \\
& \pm 9.62 & & \pm 10.42 \\
Segments & 52 & 190 & 242 \\
\hline Incidence of DO in OLF segments: $21.5 \%$ (52/242). \\
Incidence of DO in patients with OLF: $43.4 \%(23 / 53)$. \\
DO, dural ossification; OLF, ossification of ligamentum flavum.
\end{tabular}

Fifty-two segments from 23 patients with OLF had DO related to OLF. The incidence of DO in OLF segments was $21.5 \%(52 / 242)$ and the incidence of DO in patients with OLF was $43.4 \%$ (23/53; table 1$)$.

\section{Distribution}

The distribution of OLF and DO was shown in figure 3. OLF was more common in the lower thoracic spine, and more than half $(53.8 \%)$ of the DO was located in T9-T12. The majority of patients with DO (20/23, $87.0 \%$ ) had only 1-3 segments involved (figure 4).

\section{Accuracy of imaging diagnosis}

In the imaging evaluation, 60 segments were initially thought to have DO. Of them, 11 with TTS had no intraoperative evidence of DO (table 2). Nearly half of these $(5 / 11,45.5 \%)$ were adjacent to segments with DO. Only four patients without $\mathrm{DO}$ were misdiagnosed with $\mathrm{DO}$. Of 


\section{Distribution of OLF and DO}

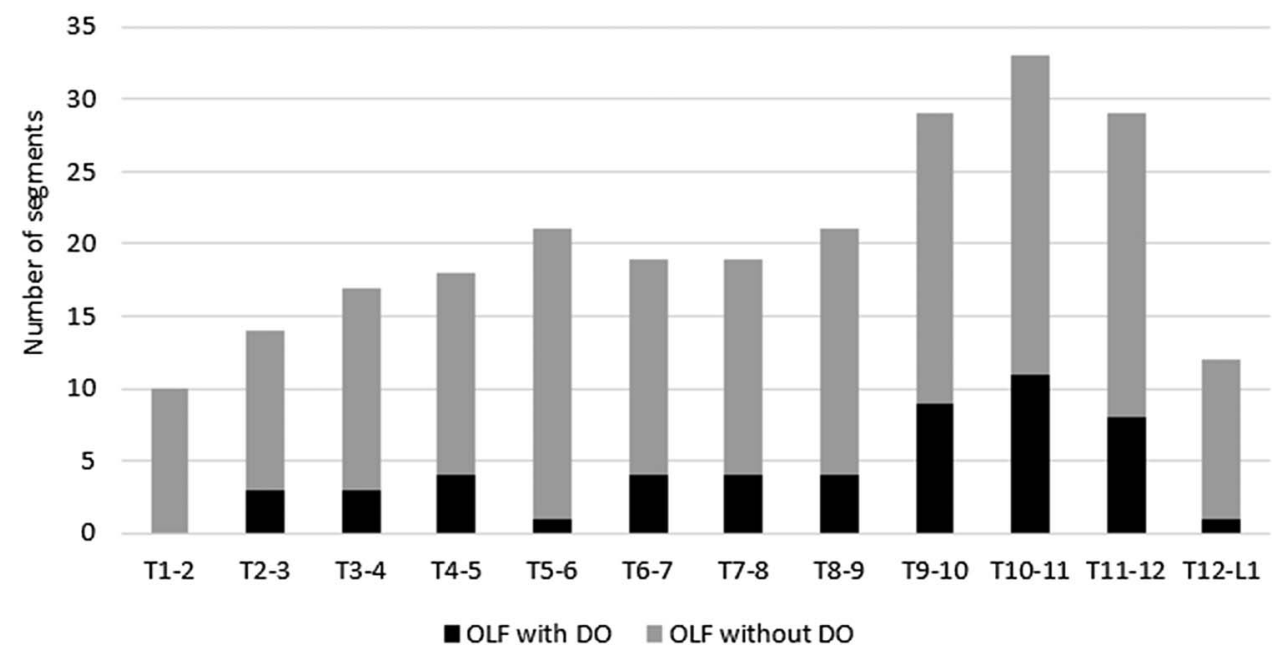

Figure 3 Distribution of OLF and DO: OLF was more common in the lower thoracic spine. More than half (53.8\%) of the DO was located in T9-T12. DO, dural ossification; OLF, ossification of ligamentum flavum.

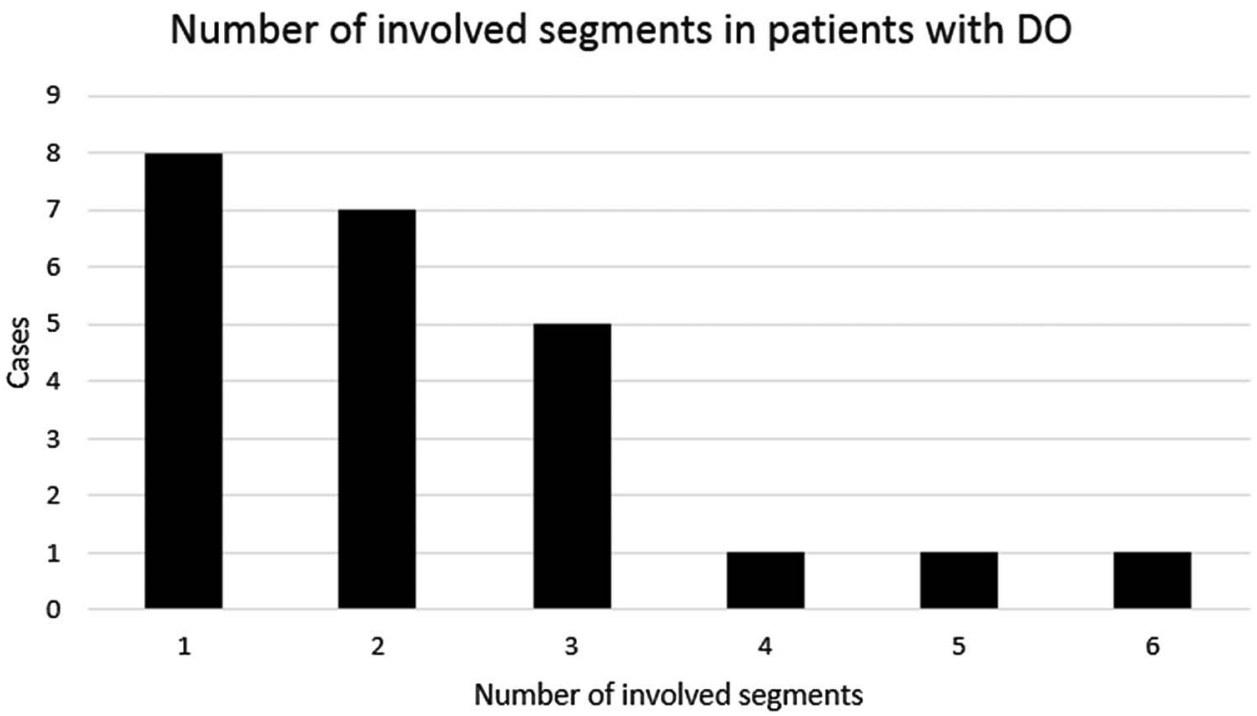

Figure 4 Number of involved segments in patients with DO: the majority (87.0\%) of patients with DO had only $1-3$ segments involving DO. DO, dural ossification.

the 52 segments with DO, 3 did not have any of the aforementioned special imaging signs. The remaining $49 \mathrm{had}$ both intraoperative evidence of $\mathrm{DO}$ and imaging findings of TTS, CS or BS. In total, $90.4 \%$ of the segments with DO had TTS. CS (13.4\%) and BS (13.4\%) could exist alone or were accompanied by TTS in the images (table 2). The sensitivity and specificity of imaging diagnosis were $94.23 \%(49 / 52)$ and $94.21 \%(179 / 190)$, respectively (table 3 ). Positive predictive value was relatively low $(81.67 \%, 49 / 60)$, due to the false positive of TTS.

\section{DISCUSSION}

As the primary cause of thoracic myelopathy, OLF commonly affects the lower thoracic spine in adults aged between 40 and 60 years. $^{2-411} 12$ This was supported by this study, which further indicated that more than half $(53.8 \%)$ of the DO was also located in T9-T12 (figure 3). The relatively high frequency of motion in this section may be one cause of this phenomenon. ${ }^{13} 14$

Since conservative treatment is ineffective, surgical decompression has become the best option for patients with OLF. $^{2-6}$ When dura mater ossifies together with ligamentum flavum, the difficulty and risks of surgery will significantly increase. In this study, the incidence of DO in patients with OLF was as high as $43.4 \%$, which was consistent with the study of Muthukumar. ${ }^{9}$ It indicated the importance of diagnosis of DO. Preoperative identification of DO can help surgeons to adopt an appropriate surgical technique and get prepared to deal with the intraoperative dural laceration. ${ }^{29}$ 
Table 2 Correlation between imaging signs and DO in OLF

\begin{tabular}{|c|c|c|c|}
\hline & OLF with DO & $\begin{array}{l}\text { OLF } \\
\text { without DO }\end{array}$ & Total \\
\hline TTS & 47 & 11 & 58 \\
\hline CS & $\begin{array}{l}7(\mathrm{CS}=1, \mathrm{CS} \\
+\mathrm{TTS}=6)^{*}\end{array}$ & 0 & $\begin{array}{l}7(\mathrm{CS}=1, \mathrm{CS} \\
+\mathrm{TTS}=6)^{*}\end{array}$ \\
\hline BS & $\begin{array}{l}7(\mathrm{BS}=1, \mathrm{BS} \\
+\mathrm{TTS}=6)^{*}\end{array}$ & 0 & $\begin{array}{l}7(\mathrm{BS}=1, \mathrm{BS} \\
+\mathrm{TTS}=6)^{*}\end{array}$ \\
\hline $\begin{array}{l}\text { No TTS, } \\
\text { CS or BS }\end{array}$ & 3 & 179 & 182 \\
\hline Total & 52 & 190 & 242 \\
\hline \multicolumn{4}{|c|}{$\begin{array}{l}{ }^{*} \text { CS/BS existed alone for one segment and was accompanied by } \\
\text { TTS in six segments. } \\
\text { BS, bridge sign; CS, comma sign; DO, dural ossification; OLF, } \\
\text { ossification of ligamentum flavum; TTS, tram track sign. }\end{array}$} \\
\hline
\end{tabular}

Table 3 Assessment of accuracy of imaging diagnosis

\begin{tabular}{lccc}
\hline & $\begin{array}{l}\text { OLF } \\
\text { with DO }\end{array}$ & $\begin{array}{l}\text { OLF } \\
\text { without DO }\end{array}$ & Total \\
\hline Segments with T/C/B & 49 & 11 & 60 \\
Segments without T/C/B & 3 & 179 & 182 \\
Total & 52 & 190 & 242 \\
\hline
\end{tabular}

Sensitivity=94.23\% (49/52), specificity=94.21\% (179/190). Positive predictive values $=81.67 \%(49 / 60)$, negative predictive values $=98.35 \%(179 / 182)$.

Test accuracy $=94.21 \%(49+179 / 242)$.

$\mathrm{B}$, bridge sign; C, comma sign; DO, dural ossification; OLF, ossification of ligamentum flavum; T, tram track sign.

It has been documented that the diagnostic value of MRI for DO associated with OLF or ossification of the posterior longitudinal ligament was inferior to $\mathrm{CT}^{8}{ }^{9}$ Therefore, an axial CT scan of surgical decompression segments was used as the primary image to be evaluated in this study. TTS, CS and BS were considered as characteristic imaging signs of DO in OLF. TTS was the most common sign among them. However, it might be misdiagnosed. In the study by Sun $e t a l,{ }^{10}$ the diagnostic specificity of TTS was only 59\%. Misdiagnosis was also pointed out by Sun $e t a l l^{2}$ but no plausible solution was proposed. In our preliminary screening study, we found that the TTS in a diagnosis of DO should be more precisely called a 'TTS between ossified ligamentum flavum and dura mater'. To improve the accuracy of diagnosis, we defined and excluded four kinds of confusing signs (false TTS; figure 2): a false TTS consisted of a facet joint (figure 2A); a false TTS consisted of an ossified ligamentum flavum and superior articular process (figure 2B); a false TTS between the ossified ligamentum flavum and lamina (figure 2C) and a false TTS between the inner and outer bone cortex of a superior articular process (figure 2D). Among them, the false TTS of the ossified ligamentum flavum and superior articular process or lamina was easily misdiagnosed as DO. ${ }^{2}$ For atypical signs, reference to adjacent imaging of the same segment sometimes could be helpful

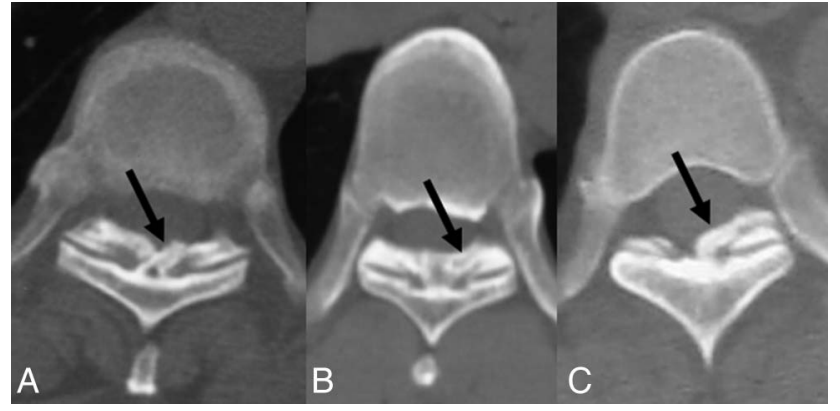

Figure 5 False-positive TTS of three segments were considered to be the imaging manifestation of incomplete ossification of ligamentum flavum (ossification of the posterior side and dural side). TTS, tram track sign.

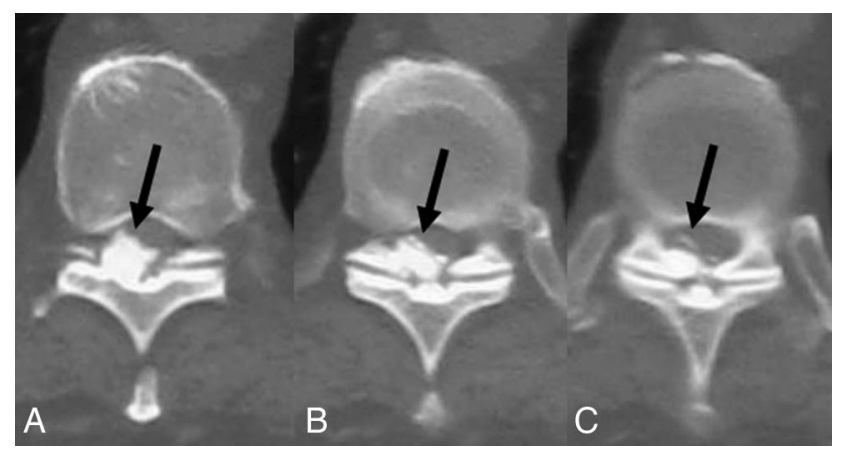

Figure 6 CT scan of the same segment: the most serious compression level $(A)$ had no TTS, while adjacent levels (B and $\mathrm{C}$ ) under less compression did.

(figure 2E). After excluding these false TTS, the specificity of imaging diagnosis was $94.21 \%(179 / 190)$ and the sensitivity remained high at $94.23 \%(49 / 52)$.

However, there were still 11 segments without DO, while having false positives of TTS. It led that the positive predictive value of imaging diagnosis was relatively low $(81.67 \%, 49 / 60)$. We further analysed these misdiagnosed segments and found that five of them were adjacent to the segments with DO. This phenomenon was previously reported in the literature. ${ }^{12}$ False-positive TTS of another three segments was considered to be the imaging manifestation of incomplete OLF (ossification of the posterior and dural side of ligamentum flavum; figure 5). This was uncommon but hard to distinguish from TTS of the ossified ligamentum flavum and dura mater. The remaining three segments were misdiagnosed for irregular signal. All the segments with BS or CS had DO.

The exact pathogenesis of OLF is still unknown. Both intrinsic $^{15-21}$ and extrinsic ${ }^{22-24}$ factors were reported to be associated with the ossification of the spinal ligament. Currently, mechanical stress as an extrinsic factor is thought to be important in the process of OLF. When the spinal ligament is under tension stress, some fibroblastic cells can transform into chondrocytes and expression of BMP-2, TGFb and SOX9 will increase. ${ }^{23} 24$ 
Yayama $e t a l^{23}$ postulated that ossification of dura mater is probably due to dilution of the above cytokines related to osteogenesis from the ossified ligamentum flavum. Based on the literature, a potential mechanism of DO was proposed before. ${ }^{14}$ The relative movement between OLF and compressed dura mater, due to the flexion and extension of the spine, will cause local inflammation, leading to dural adhesion. ${ }^{14}$ Then the adhesion tissue can act as a tunnel to transport osteogenic cytokines aforementioned from OLF to compressed dura mater, which causes the dura mater directly adhered to OLF first ossifies. ${ }^{14}$ According to this, DO may start at the portion that is directly in contact with the ossified ligamentum flavum. Since there is no gap between the ossified dura mater and ligamentum flavum, no special imaging signs will appear. This could explain why some segments with DO did not have any of the aforementioned special imaging signs in previous studies ${ }^{910}$ and ours. Only when the ossification extended to the surrounding dura mater that was not in contact with the ossified ligamentum flavum, these special imaging findings would appear. In the same segment CT scan of some patients, the most serious compression level did not have these special imaging findings, while adjacent levels under less compression did (figure 6). This phenomenon was a supporting evidence of our hypothesis.

This study made a comprehensive analysis of DO. Radiological characteristics of DO were described and analysed in detail. Limitations still exist in this study. The study population comprised patients with OLF who needed surgical treatment. So the incidence and distribution of DO reported in this study was restricted to this population. The mechanism and progression of DO need to be further investigated in the future. Large sample multicentre prospective studies are still needed to further assess the accuracy of imaging diagnosis.

\section{CONCLUSIONS}

DO was relatively common in thoracic OLF. T9-T12 were the most frequently involved segments. TTS might be misdiagnosed. After excluding four kinds of false TTS, the accuracy of imaging diagnosis was relatively high.

\section{Author affiliations \\ ${ }^{1}$ Department of Orthopaedic Surgery, Peking Union Medical College Hospital, Chinese Academy of Medical Sciences and Peking Union Medical College, Beijing, China \\ ${ }^{2}$ Department of Trauma \& Orthopaedic Surgery, Hillingdon Hospital, London, UK}

Contributors $Y Z, B L, G Q$ and $S G$ were involved in study design. $Y Z, B L, W L$, $\mathrm{YL}, \mathrm{HP}$ and $\mathrm{CW}$ were involved in data acquisition. $\mathrm{YZ}, \mathrm{BL}, \mathrm{SG}, \mathrm{WL}$ and $\mathrm{YL}$ were involved in analysis and interpretation of data.

Funding This work was supported by grants from the National Natural Science Foundation of China (grant number 81572093) and Beijing Natural Science Foundation (7162153).

Competing interests None declared.
Ethics approval The Institutional Review Board (IRB) of Peking Union Medical College Hospital (PUMCH).

Provenance and peer review Not commissioned; externally peer reviewed.

Data sharing statement No additional data are available.

Open Access This is an Open Access article distributed in accordance with the Creative Commons Attribution Non Commercial (CC BY-NC 4.0) license, which permits others to distribute, remix, adapt, build upon this work noncommercially, and license their derivative works on different terms, provided the original work is properly cited and the use is non-commercial. See: http:// creativecommons.org/licenses/by-nc/4.0/

\section{REFERENCES}

1. Sato T, Kokubun S, Tanaka Y, et al. Thoracic myelopathy in the Japanese: epidemiological and clinical observations on the cases in Miyagi Prefecture. Tohoku J Exp Med 1998;184:1-11.

2. Sun J, Zhang C, Ning G, et al. Surgical strategies for ossified ligamentum flavum associated with dural ossification in thoracic spinal stenosis. J Clin Neurosci 2014;21:2102-6.

3. Aizawa $\mathrm{T}$, Sato $\mathrm{T}$, Sasaki $\mathrm{H}$, et al. Thoracic myelopathy caused by ossification of the ligamentum flavum: clinical features and surgical results in the Japanese population. J Neurosurg Spine 2006;5:514-19.

4. Inamasu J, Guiot BH. A review of factors predictive of surgical outcome for ossification of the ligamentum flavum of the thoracic spine. J Neurosurg Spine 2006;5:133-9.

5. Miyakoshi N, Shimada Y, Suzuki T, et al. Factors related to long-term outcome after decompressive surgery for ossification of the ligamentum flavum of the thoracic spine. J Neurosurg 2003;99(3 Suppl):251-6.

6. Pascal-Moussellard H, Cabre P, Smadja D, et al. Symptomatic ossification of the ligamentum flavum: a clinical series from the French Antilles. Spine 2005;30:E400-5.

7. Sun X, Sun C, Liu X, et al. The frequency and treatment of dural tears and cerebrospinal fluid leakage in 266 patients with thoracic myelopathy caused by ossification of the ligamentum flavum. Spine 2012;37:E702-7.

8. Mizuno J, Nakagawa $\mathrm{H}$, Matsuo $\mathrm{N}$, et al. Dural ossification associated with cervical ossification of the posterior longitudinal ligament: frequency of dural ossification and comparison of neuroimaging modalities in ability to identify the disease. J Neurosurg Spine 2005;2:425-30.

9. Muthukumar N. Dural ossification in ossification of the ligamentum flavum: a preliminary report. Spine 2009;34:2654-61.

10. Sun XZ, Chen ZQ, Qi Q, et al. Diagnosis and treatment of ossification of the ligamentum flavum associated with dural ossification: clinical article. J Neurosurg Spine 2011;15:386-92.

11. Guo JJ, Luk KD, Karppinen J, et al. Prevalence, distribution, and morphology of ossification of the ligamentum flavum: a population study of one thousand seven hundred thirty-six magnetic resonance imaging scans. Spine 2010;35:51-6.

12. Yang $Z$, Xue $Y$, Zhang $C$, et al. Surgical treatment of ossification of the ligamentum flavum associated with dural ossification in the thoracic spine. J Clin Neurosci 2013;20:212-16.

13. Xu P, Wang $\mathrm{C}, \mathrm{He} \mathrm{H}$, et al. Spinal balance failure: a potential cause of spinal ligament ossification. Med Hypotheses 2011;76:908-10.

14. Li B, Guo S, Qiu G, et al. A potential mechanism of dural ossification in ossification of ligamentum flavum. Med Hypotheses 2016;92:1-2.

15. Goto K, Yamazaki M, Tagawa M, et al. Involvement of insulin-like growth factor I in development of ossification of the posterior longitudinal ligament of the spine. Calcif Tissue Int 1998;62:158-65.

16. Akune T, Ogata N, Seichi A, et al. Insulin secretory response is positively associated with the extent of ossification of the posterior longitudinal ligament of the spine. J Bone Joint Surg Am 2001;83-A:1537-44.

17. Shingyouchi Y, Nagahama A, Niida M. Ligamentous ossification of the cervical spine in the late middle-aged Japanese men. Its relation to body mass index and glucose metabolism. Spine 1996;21:2474-8.

18. Li H, Jiang LS, Dai LY. Hormones and growth factors in the pathogenesis of spinal ligament ossification. Eur Spine $J$ 2007;16:1075-84

19. Maeda S, Koga H, Matsunaga S, et al. Gender-specific haplotype association of collagen alpha2 (XI) gene in ossification of the posterior longitudinal ligament of the spine. J Hum Genet 2001;46:1-4. 
20. Nakamura I, Ikegawa S, Okawa A, et al. Association of the human NPPS gene with ossification of the posterior longitudinal ligament of the spine (OPLL). Hum Genet 1999;104:492-7.

21. Yamauchi T, Taketomi E, Matsunaga S, et al. Bone mineral density in patients with ossification of the posterior longitudinal ligament in the cervical spine. J Bone Miner Metab 1999;17:296-300.

22. Kawaguchi $\mathrm{Y}$, Kanamori $\mathrm{M}$, Ishihara $\mathrm{H}$, et al. Progression of ossification of the posterior longitudinal ligament following en bloc cervical laminoplasty. J Bone Joint Surg Am 2001;83-A:1798-802.
23. Yayama T, Uchida K, Kobayashi S, et al. Thoracic ossification of the human ligamentum flavum: histopathological and immunohistochemical findings around the ossified lesion. J Neurosurg Spine 2007;7: 184-93.

24. Tsukamoto $\mathrm{N}$, Maeda $\mathrm{T}$, Miura $\mathrm{H}$, et al. Repetitive tensile stress to rat caudal vertebrae inducing cartilage formation in the spinal ligaments: a possible role of mechanical stress in the development of ossification of the spinal ligaments. J Neurosurg Spine 2006:5:234-42. 